\title{
The Analysis of Morphoradiological Parameters in Predicting Risk of Basilar Artery Tip Aneurysm Rupture: A Retrospective Cohort Study
}

\author{
Abdulaziz Al-Sharydah (1D ${ }^{\prime}$ \\ Abdulrahman \\ Al-Abdulwahhab (D) \\ Sari Al-Suhibani $\mathbb{D}^{\prime}$ \\ Afnan Al-Muhanna (D) \\ Abdullah Abohimed (D) ${ }^{2}$ \\ Abdulmonem AlSharidah (iD) ${ }^{3}$ \\ Faisal Alabbas (D) $^{3}$ \\ 'Diagnostic and Interventional Radiology \\ Department, Imam Abdulrahman Bin \\ Faisal University, King Fahd Hospital of \\ the University, Al-Khobar City, Eastern \\ Province, Saudi Arabia; ${ }^{2}$ Department of \\ Medical Imaging, Ministry of National \\ Guard Health Affairs, King Abdulaziz \\ Medical City, Riyadh, Saudi Arabia; \\ ${ }^{3}$ College of Medicine, Imam Abdulrahman \\ Bin Faisal University, King Fahd Hospital \\ of the University, Al-Khobar City, Eastern \\ Province, Saudi Arabia
}

\begin{abstract}
Purpose: Basilar artery tip aneurysms can have fatal consequences and poor prognostic outcomes in case of rupture. We investigated the standard parameters used to evaluate aneurysmal morphology to predict aneurysm rupture.

Patients and Methods: We measured the differences between ruptured and unruptured basilar bifurcation aneurysms in terms of morphological features, including aneurysm size, size ratio, aneurysm height, perpendicular height, aneurysm width, bottleneck ratio, aspect ratio, and present daughter domes, using 3D angiography.

Results: Among 34 patients, 21 (61.8\%) and 13 (38.2\%) had ruptured and unruptured basilar tip aneurysms, respectively. In the ruptured group, the mean aneurysm size, and aspect, size, and bottleneck ratios were $6.8 \pm 1.9 \mathrm{~mm}, 3.2 \pm 1.0,2.3 \pm 0.5$, and $2.2 \pm 0.4$, respectively, compared to $4.5 \pm 1.5 \mathrm{~mm}, 4.4 \pm 2.0,1.6 \pm 0.4$, and $2.7 \pm 0.7$, respectively, in the non-ruptured group. Univariate analysis showed a larger aneurysmal cross-sectional diameter (odds ratio $[\mathrm{OR}], 0.50 ; 95 \%$ confidence interval $[\mathrm{CI}], 0.5-5.392 ; \mathrm{p}<0.05$ ), a larger size ratio (OR, 21.375; 95\% CI, 3.283-139.177; $\mathrm{p}<0.05)$, and presence of a daughter dome $(\mathrm{OR}, 72.0 ; 95 \% \mathrm{CI}, 6.7-776.5 ; \mathrm{p}<0.05)$ with ruptured basilar artery tip aneurysms.
\end{abstract}

Conclusion: A larger aneurysm size and size ratio, and the presence of a daughter dome were significantly associated with increased rupture risk in basilar tip aneurysms.

Keywords: aneurysm, angiography, brain, basilar artery, hemorrhage

\section{Introduction}

Subarachnoid hemorrhage is a life-threatening complication of ruptured intracranial aneurysms. ${ }^{1}$ Basilar artery (BA) aneurysms may occur at several locations, including the BA junctions with the vertebral, anterior inferior cerebellar, and superior cerebellar arteries, and most commonly, before the bifurcation into posterior cerebral arteries. ${ }^{2}$ BA tip aneurysms have a higher tendency to rupture compared to other cerebral aneurysms. ${ }^{2}$

Unruptured basilar tip aneurysms account for nearly 3\% of all intracranial aneurysms. ${ }^{3}$ Morphological analyses have shown that aneurysmal geometric parameters, including aneurysm size (AS), size ratio (SR), aneurysm height (AH), perpendicular height $(\mathrm{PH})$, aneurysm width $(\mathrm{AW})$, bottleneck ratio (BNR), aspect ratio (AR), and presence of daughter dome (DD), can predict rupture. ${ }^{4,5}$ Computational fluid dynamics studies have an essential role in assessing hemodynamic factors to predict aneurysm progression and rupture. ${ }^{6} \mathrm{CT}$ angiography (CTA) Al-Abdulwahhab

Diagnostic and Interventional Radiology Department, Imam Abdulrahman Bin Faisal University, King Fahd Hospital of the University, P.O. Box: 31952 (4398), AlKhobar City, Eastern Province, Saudi Arabia Tel +9665023337I I

Email ahAbdulwahab@iau.edu.sa 
is the primary diagnostic tool to provide anatomic and morphological features of intracranial aneurysms. ${ }^{7}$ Zhang et $\mathrm{al}^{8}$ revealed DD presence, AR, and larger flow angle to be associated with BA tip aneurysm rupture. Most recently, a suggested simple machine-based nomogram based on machine learning constructed to predict aneurysmal rupture risk at different locations achieved accurate results. ${ }^{8}$

In this study, we performed a retrospective analysis of patients with BA tip aneurysms and compared and analyzed the significance of eight morphological parameters between ruptured and unruptured aneurysm groups using reconstructed 3D rotational angiograms.

\section{Materials and Methods}

\section{Setting}

The study was conducted at the King Fahad Hospital of the University, a Joint Commission Internationalaccredited, tertiary-care, university teaching hospital with 700 beds in the eastern province of Saudi Arabia. The Interventional Radiology division of the Radiology Department constitutes four modern angiography suites.

\section{Study Design, Patient Inclusion and Exclusion}

In a retrospective cohort study from October 2013 to September 2019, 34 patients with BA tip aneurysms were identified using the electronic medical record system and confirmed by CTA. We defined the BA tip as the distal point before the bifurcation into the posterior cerebral arteries. The aneurysms were then classified as either ruptured $(n=21)$ or unruptured $(n=13)$, according to initial CT findings on admission. We excluded fusiform, dissecting, vascular malformation-associated, non-BA, and CTA-indeterminate types of aneurysms.

\section{Definition of Morphological Parameters}

Using 3D reconstructions of CTA data, we measured eight morphological parameters as follows: AS, using the maximum aneurysmal cross-sectional diameter; $\mathrm{AH}$, using the maximum distance to the aneurysm dome from the halfway of the neck; PH, using the maximum vertical distance from the center of the neck to the dome; AW, using the maximum distance perpendicular to the height; BNR, by calculating the ratio between maximum width and neck size; AR, by calculating the ratio between vertical height and neck size; SR, by calculating the ratio between aneurysm height and mean parent vessel size; and DD, using the bulging sac at the aneurysm dome. We defined the mean vessel size as the average cross-sectional diameter of the parent artery close to the aneurysm neck.

\section{CTA}

The morphological parameters were assessed by two independent board-certified neuroradiologists and two interventional radiologists with more than 5 years of experience on CTA image reconstructions using Syngo MMWP (VE32D, Siemens Healthcare, Forchheim, Germany) on a standard post-processing workstation. Images were acquired using a 64-channel multidetector CT scanner and a 128-slice dualsource CT system (Somatom Definition FLASH, Siemens Healthcare, Forchheim, Germany).

Brain CTA was performed in our hospital using a helical method, scanning from the aortic arch to the skull vertex. The imaging parameters were as follows: voltage, 120-140 kV; current, 150-200 mA; noise index, 9; adaptive statistical iterative reconstruction, $40 \%$; collimation, $64 \times 2 \times 0.6 \mathrm{~mm}^{3}$; rotation time, $0.28 \mathrm{~s}$; and pitch factor, 1.2. All images were reconstructed with a $1.0-\mathrm{mm}$ slice thickness, $0.9-\mathrm{mm}$ slice interval, 512-pixel image matrix, and 236-286-mm adapted field of view. A contrast agent $(70-100 \mathrm{~mL}$ of Ultravist 300 ; Schering, Berlin, Germany) was administered through the left antecubital vein at a flow rate of 5-6 mL/s via an 18-22gauge cannula using a dual-head power injector (Empower, EZEM Inc., New York, USA), followed by a $50-\mathrm{mL}$ saline flush. We used a bolus tracking method that measures the attenuation values within an internal carotid artery to achieve optimal synchronization of the contrast medium flow. Once the attenuation values exceeded the threshold of 100-120 Hounsfield units, automatic caudocranial scanning was initiated.

\section{Statistical Analysis}

We interpreted and analyzed the data using IBM SPSS (version 22.0; IBM SPSS, Armonk, New York, USA). We used univariate logistic regression analysis to determine potential variables associated with ruptured aneurysms and compared them to the unruptured group. All values were expressed as means \pm standard deviations, and statistical significance was set at $\mathrm{p}<0.05$.

\section{Ethical Approval}

This retrospective study was approved by the local institutional review board of Imam Abdulrahman Bin Faisal University (IRB-2019-01-400). Given the purely 
Table I Demographic Data and Clinical Risk Factors of Patients with Ruptured and Unruptured Basilar Tip Aneurysms

\begin{tabular}{|c|c|c|c|c|}
\hline Variables & $\begin{array}{l}\text { All Patients } \\
\mathbf{N}=\mathbf{3 4}\end{array}$ & $\begin{array}{l}\text { Ruptured Aneurysms } \\
N=2 \text { I }\end{array}$ & $\begin{array}{l}\text { Unruptured Aneurysms } \\
N=13\end{array}$ & $P$ value \\
\hline Age (years), mean \pm SD & $57.0 \pm 13.0$ & $42.1 \pm 11$ & $59.4 \pm 14$ & 0.04 \\
\hline Female, n (\%) & $22(64.7)$ & $15(7 \mid .4)$ & $7(53.8)$ & 0.70 \\
\hline Smoking, n (\%) & $24(70.5)$ & $17(80.9)$ & $8(61.5)$ & 0.74 \\
\hline Hypertension, n (\%) & $20(58.8)$ & $13(61.9)$ & $7(53.8)$ & 0.58 \\
\hline Type 2 diabetes mellitus, $n(\%)$ & $17(50)$ & II (52.3) & $6(46.1)$ & 0.64 \\
\hline Dyslipidemia, n (\%) & $10(29.4)$ & $8(38.1)$ & $2(15.3)$ & 0.72 \\
\hline Family history of SAH (first-degree), n (\%) & $2(5.88)$ & $2(9.5)$ & $0(0)$ & 0.97 \\
\hline Family history of aneurysms, $\mathrm{n}(\%)$ & $5(14.7)$ & $3(14.2)$ & $2(15.3)$ & 0.86 \\
\hline
\end{tabular}

descriptive and retrospective nature of the study and in compliance with the Declaration of Helsinki, informed written consent was provided by each participant. Data were collected anonymously and reported only in aggregate form.

\section{Results}

\section{Baseline Analysis}

Of 34 patients, ruptured and unruptured BA tip aneurysms were seen in $21(61.8 \%)$ and $13(38.2 \%)$ patients, respectively. The mean age was $55.9 \pm 18.3$ years (range, 17-89 years), and $25(73.5 \%)$ patients were female. Patients in the ruptured group were frequently older, with a mean age of $60.8 \pm 17.5$ years, compared to $48.0 \pm 17.4$ years for patients in the unruptured group $(\mathrm{p}=0.047)$. In the ruptured group, $16(76.2 \%)$ were female, compared to 9 $(69.2 \%)$ in the unruptured group, with no significant difference in sex between the two groups $(p=0.704)$. Tobacco use was the most common risk factor among both groups $(\mathrm{p}=0.74)($ Table 1$)$.

\section{Morphological Analysis}

The mean AS, AR, SR, and BNR in the ruptured group were $6.8 \pm 1.9 \mathrm{~mm}, 3.2 \pm 1.0,2.3 \pm 0.5$, and $2.2 \pm 0.4$, respectively, compared to $4.5 \pm 1.5 \mathrm{~mm}, 4.4 \pm 2.0,1.6 \pm$ $0.4,2.7 \pm 0.7$, respectively, in the unruptured group (Table 2). Univariate analysis showed that a larger $\mathrm{AH}$ and AW, indicating a larger overall cross-sectional

Table 2 BA Tip Aneurysm Characteristics Stratified by Rupture Status

\begin{tabular}{|c|c|c|c|c|}
\hline Variables & $\begin{array}{l}\text { All Patients } \\
\mathbf{N}=\mathbf{3 4}\end{array}$ & $\begin{array}{l}\text { Ruptured Aneurysms } \\
\mathbf{N}=2 \text { I }\end{array}$ & $\begin{array}{l}\text { Unruptured Aneurysms } \\
N=13\end{array}$ & $P$ value \\
\hline Daughter dome (\%) & $15(44.1)$ & $13(61.9)$ & I (7.6) & $<0.05$ \\
\hline Posterior projection (\%) & $22(64.7)$ & $13(61.9)$ & $8(6 \mid .5)$ & 0.26 \\
\hline Maximum aneurysm height $(\mathrm{mm})$, mean $\pm \mathrm{SD}$ & $7.2 \pm 2.5$ & $6.16 \pm 3.1$ & $4.32 \pm 2.1$ & 0.85 \\
\hline Perpendicular height $(\mathrm{mm})$, mean $\pm \mathrm{SD}$ & $6.78 \pm 3.3$ & $6.24 \pm 1.8$ & $5.84 \pm 2.4$ & $<0.05$ \\
\hline Neck diameter $(\mathrm{mm})$, mean $\pm \mathrm{SD}$ & $3.65 \pm 1.4$ & $3.12 \pm 1.9$ & $3.44 \pm 1.7$ & 0.17 \\
\hline Maximum aneurysm width $(\mathrm{mm})$, mean $\pm \mathrm{SD}$ & $6.72 \pm 3.8$ & $6.55 \pm 3$ & $5.21 \pm 2.3$ & $<0.05$ \\
\hline Aspect ratio, mean $\pm S D$ & $1.25 \pm 0.59$ & $1.32 \pm 0.65$ & $1.12 \pm 0.42$ & 0.64 \\
\hline Parent /Basilar artery diameter $(\mathrm{mm})$, mean $\pm \mathrm{SD}$ & $2.750 \pm 0.41$ & $2.63 \pm 0.53$ & $2.48 \pm 0.385$ & 0.35 \\
\hline Size ratio, mean \pm SD & $1.23 \pm 0.73$ & $1.14 \pm 0.66$ & $1.09 \pm 0.62$ & $<0.05$ \\
\hline Bottle neck ratio, mean $\pm S D$ & $\mathrm{I} . \mathrm{I} \pm 0.44$ & $0.98 \pm 0.51$ & $0.75 \pm 0.54$ & 0.321 \\
\hline
\end{tabular}



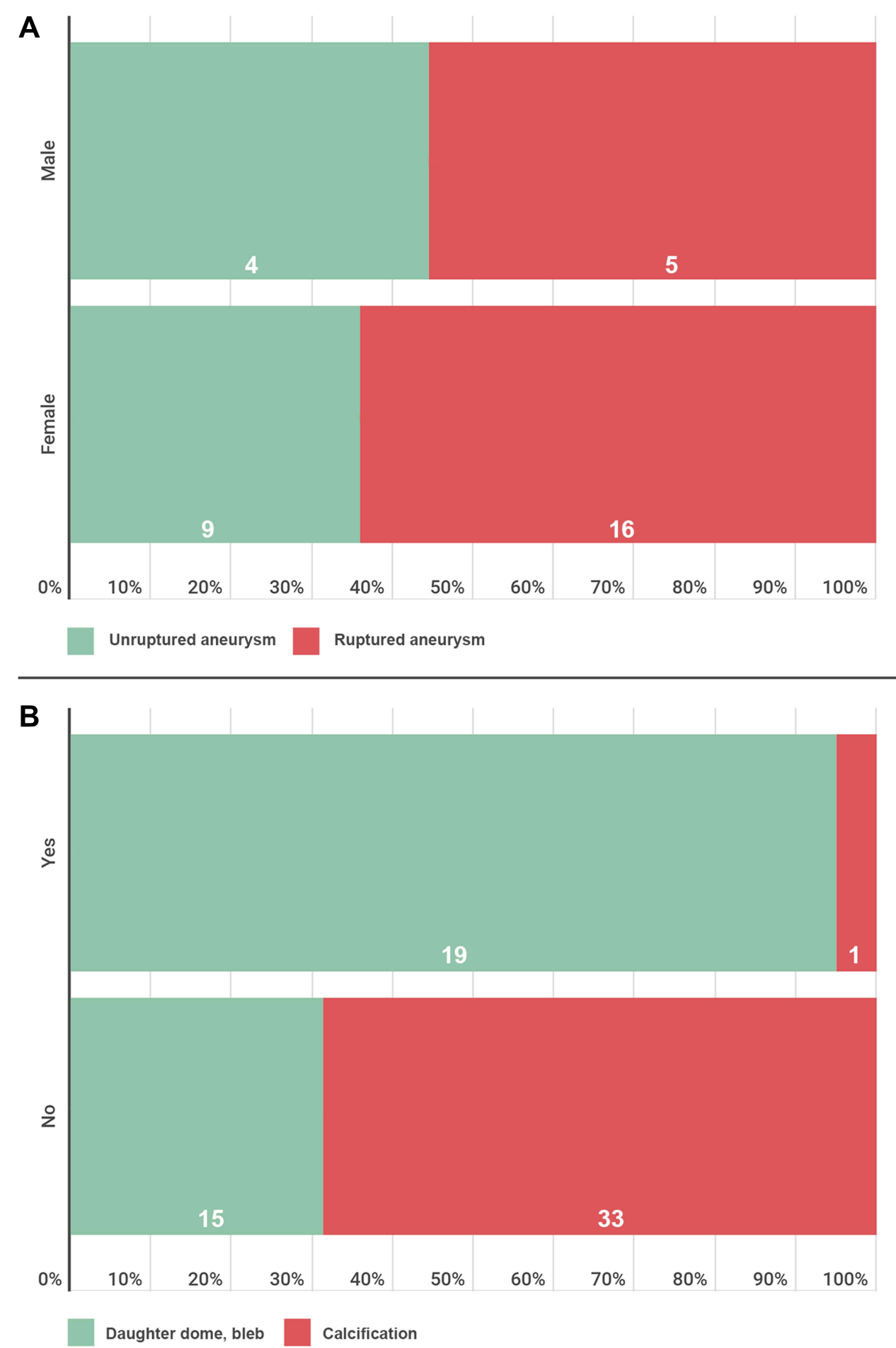

Figure I Stacked bar histograms illustrating the following: (A) patient demographics and aneurysmal hemodynamic status, and (B) morpho-radiological characteristics of the patient under investigation.

\section{diameter (odds ratio [OR], 0.50; 95\% confidence interval Discussion}

[CI], 0.5-5.392, $\mathrm{p}<0.05)$, larger SR (OR, 21.375, 95\% CI, Morphological and hemodynamic differences between rup3.283-139.177, $\mathrm{p}<0.05)$, and DD (OR, 72.0; 95\% CI, tured and unruptured aneurysms have been identified and $6.7-776.5 ; \mathrm{p}<0.05)$ were associated with BA aneurysm construed. $^{8-13}$ Through our research, we noticed that bifurcarupture (Figures 1-4). tion aneurysms showed less variability in their morphological 


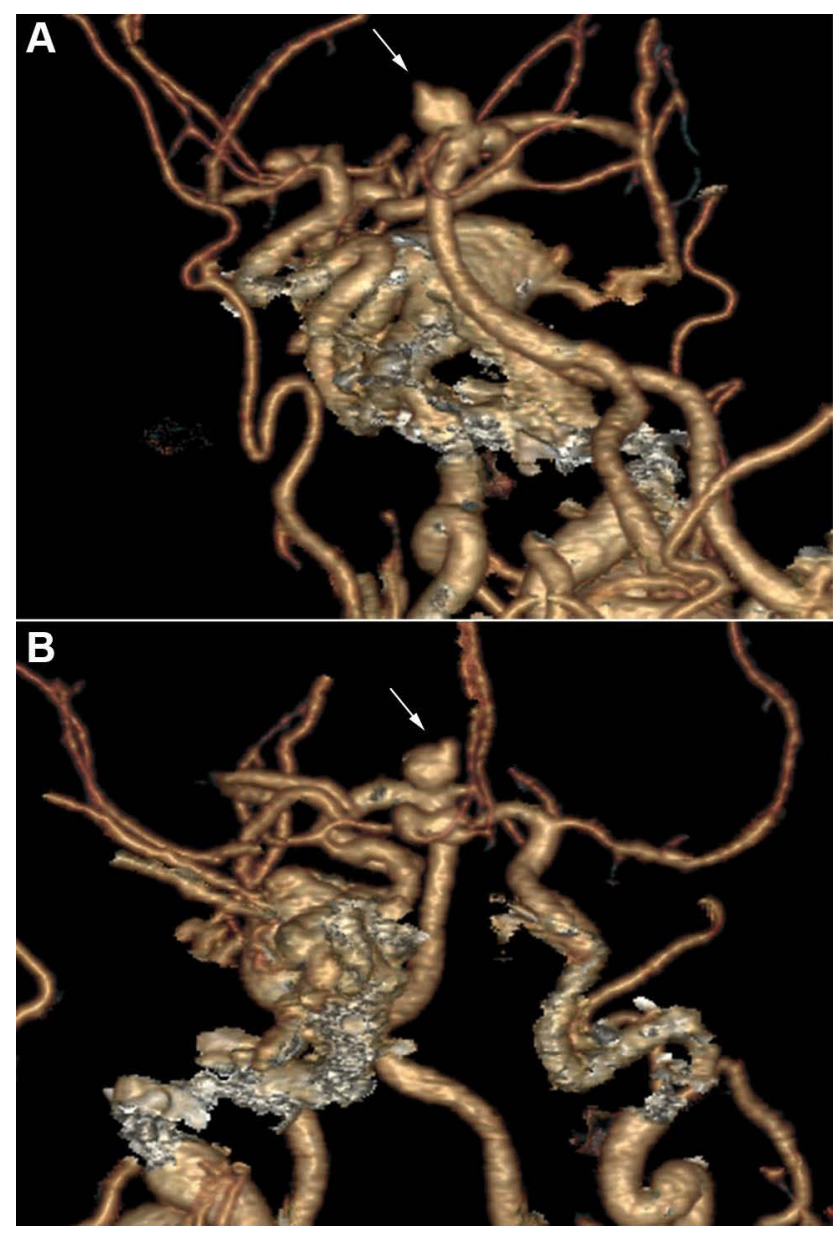

Figure 2 Ruptured basilar tip aneurysm. 3D CT angiography, (A) frontal and (B) lateral projections, shows a unilocular saccular aneurysm arising from the basilar tip (arrows) with a daughter sac at the left side of the aneurysm dome. The aneurysm is slightly inclined anteriorly. There is also an incidental finding of bilateral carotid cavernous fistulae.

characteristics and a higher predictive ability for rupture compared to sidewall aneurysms. ${ }^{11}$ Moreover, basilar bifurcation aneurysms have developed a higher prevalence of rupture than middle cerebral and internal carotid bifurcation aneurysms. This could be attributed to multiple factors, including BA embryological development, pulsatile blood flow, structural differences, and environmental effects during aneurysm development. ${ }^{14}$

This study is representative of an analytical measurement of a rare intracranial aneurysm involving the BA tip, fulfilling the gaps in related literature. It was conducted in a teaching hospital and performed by a licensed radiologist with adequate experience with aneurysm diagnoses. Furthermore, some of the authors are interventional radiologists with adequate experience in aneurysm management. Similar studies have investigated the association of only a few aneurysmal measures with rupture risk. In

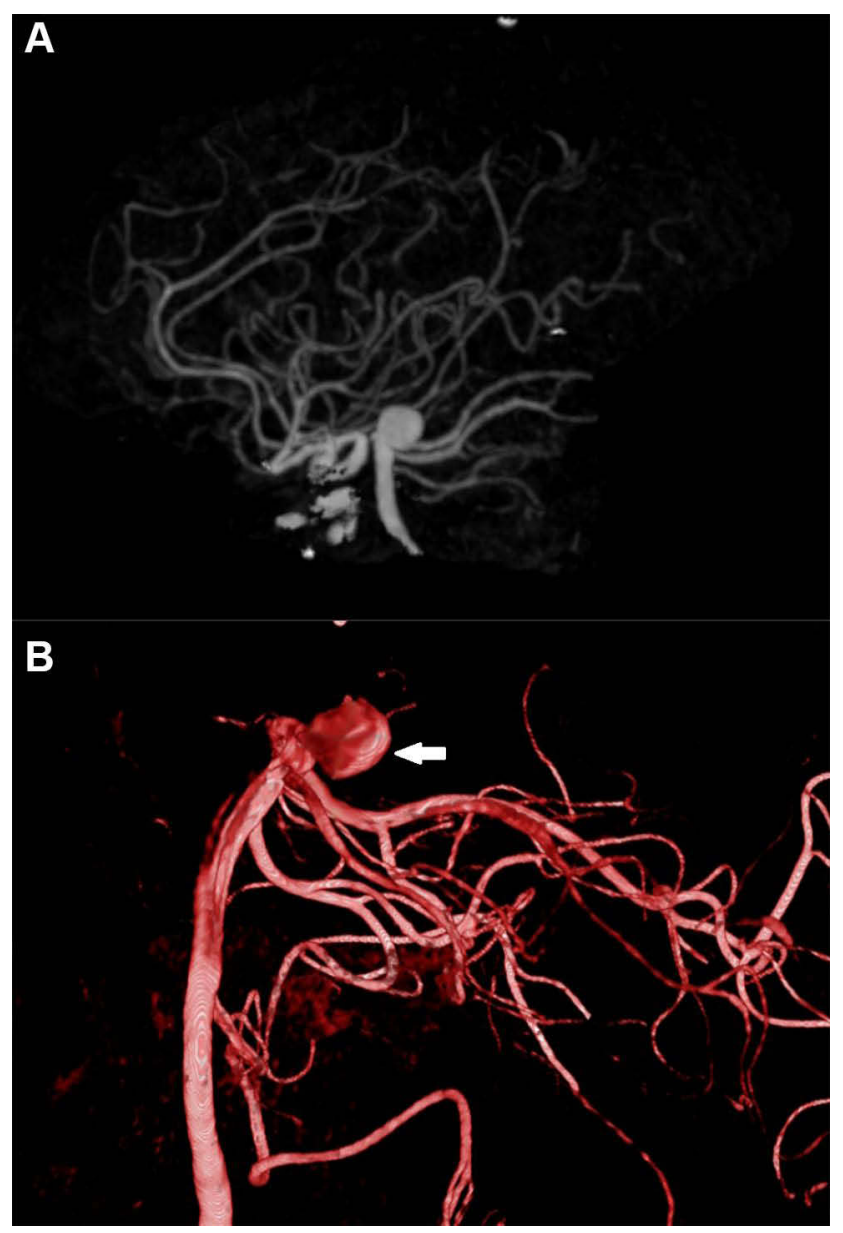

Figure 3 (A) Hemodynamically stable basilar tip aneurysm. The aneurysm is inclined posteriorly with smooth aneurysmal dome. (B) 3D lateral angiogram shows a unilocular saccular aneurysm arising from the basilar tip with posterior inclination (arrow).

contrast, our study investigated the influence of all rupture risk determinants in formulating an appropriate management plan using an unbiased and comprehensive methodology.

Wang et al ${ }^{15}$ recently examined a cohort of over 1500 Chinese patients and found that irrespective of diameter, a saccular irregularity and higher AR were associated with aneurysm rupture. In addition, the majority of ruptured intracranial aneurysms were $<7 \mathrm{~mm}$ in size. In our study, we found that a larger AS, SR, AR, BNR, and DD were independently associated with a higher risk of BA tip aneurysm rupture.

Our results showed that AR is one of the most important parameters associated with aneurysm rupture. Despite other morphological parameters that were significantly associated with increased rupture risk in BA tip aneurysms in our study, AR has been considered as the only pertinent parameter in assessing aneurysm rupture risk. 




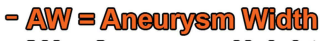

- AN a Aneurysm Heights

- PH a Aneurysm Porpendicular Melight

- DD e Daughter Dome

- SR a Sizz Ratio

- AR = Aspost Rिarto

- BNR a Bottleneck Ratio

- AS = Aneurysm size

\section{$-A S=(A W X \mathbb{A})$}

- SR a $\frac{A N H}{\text { Average parent artery size }}$

- BNR = $\frac{\text { AWV }}{\text { Aneunysm neck sizo }}$

- AR a $\frac{\text { PU }}{\text { Anevrysma neck siżo }}$

Figure 4 Schematic diagram illustrating calculation of BA aneurysm measurements.

An SR $>2$ has caused the production of complex flow patterns and low wall shear stress, leading to aneurysm rupture, particularly in basilar and middle cerebral bifurcation aneurysms. ${ }^{8,9,16}$ However, Sato et $\mathrm{al}^{17}$ reported that a larger SR is not significant factor in predicting BA tip aneurysm rupture.

A larger BNR may affect the intra-aneurysmal flow velocity by reducing intra-dome flow and shear stress, 
ultimately increasing the risk for aneurysm rupture. ${ }^{18} \mathrm{We}$ also found a significant difference in BNR between ruptured and unruptured aneurysms.

Zhang et $\mathrm{al}^{8}$ showed that the presence of DD was significantly associated with aneurysm rupture. The parent-daughter angle ratio was significantly inversely associated with ruptured basilar tip aneurysms. ${ }^{8}$ In our study, the ruptured group showed $2.5 \times$ as much DDs as those in the unruptured group ( $30.9 \%$ vs $12.3 \%$, respectively). The development of aneurysmal daughter blebs may lead to a ubiquitous intra-aneurysmal pressure, induced weak local protrusions in the aneurysm walls, and progressive development to a high rupture risk. ${ }^{19}$

Indirect signs of instability in the aneurysm walls prior to rupture have been seen in MRI scans. ${ }^{20,21}$ Flow pattern visualization and hemodynamic measurements of the intracranial aneurysm by 3D and 4D high-resolution black-blood T1-weighted volumetric isotropic turbo spin echo sequencing, which has inherent blood flow suppression before and after contrast injection, predict intracranial aneurysm rupture risk by identifying arterial wall enhancement, reflecting the processes of inflammation before rupture. ${ }^{20}$

This study had several limitations. First, our sample size was small due to the relative rarity of this type of aneurysm. Second, clinical factors were not considered in determining overall BA aneurysm rupture risk, because of limited available data in the system. Finally, aneurysmal morphology was altered due to associated subarachnoid hemorrhage and resultant vasospasm to the parent vessel and the aneurysm. Nevertheless, this study contributes to the current understanding of the factors associated with aneurysm rupture.

\section{Conclusion}

The BA tip aneurysm morphological features were analyzed using the best image projections of the aneurysm from reconstructed $3 \mathrm{D}$ rotational angiography to determine the parameters associated with the risk of aneurysm rupture. A larger AS and SR, as well as the presence of a DD, increased the risk of rupture. Further studies assessing hemodynamic flow with morphological parameters to quantify the risk of BA tip aneurysm rupture are recommended. It should be possible to implement volumetric analysis of angiographic BA aneurysms, including their anatomy and relation to the parent and adjacent vessels, to provide further information and allow fast and safe decision-making regarding the feasibility of endovascular or surgical treatment.

\section{Abbreviations}

$\mathrm{AH}$, aneurysm height; AR, aspect ratio; AS, aneurysm size; AW, aneurysm width; BA, basilar artery; BNR, bottleneck ratio; CI, confidence interval; CTA, computed tomography angiography; DD, daughter dome; OR, odds ratio; $\mathrm{PH}$, perpendicular height; SR, size ratio.

\section{Data Sharing Statement}

The corresponding author is responsible for sharing data publicly upon request from the publishing journal.

\section{Ethics Approval}

This study was approved by the institutional review board of the Imam Abdulrahman Bin Faisal University (IRB2019-01-400).

\section{Consent for Publication}

Consent for publication of the manuscript was obtained from the King Fahd Hospital of the University and the Imam Abdulrahman Bin Faisal University.

\section{Author Contributions}

All authors made a significant contribution to the work reported, whether that is in the conception, study design, execution, acquisition of data, analysis and interpretation, or in all these areas; took part in drafting, revising or critically reviewing the article; gave final approval of the version to be published; have agreed on the journal to which the article has been submitted; and agree to be accountable for all aspects of the work.

\section{Disclosure}

The authors declare that they have no conflicts of interest. This study did not receive any specific grant from funding agencies in the public, commercial, and not-for-profit sectors.

\section{References}

1. Brisman JL, Song JK, Newell DW. Cerebral aneurysms. $N$ Engl $J$ Med. 2006;355(9):928-939. doi:10.1056/NEJMra052760

2. Mori K, Toyooka T, Otani N. Surgery of upper basilar artery aneurysm. In: July J, Wahjoepramono E, editors. Neurovascular Surgery. Singapore: Springer; 2019:141-147.

3. Vlak MH, Algra A, Brandenburg R, Rinkel GJ. Prevalence of unruptured intracranial aneurysms, with emphasis on sex, age, comorbidity, country, and time period: a systematic review and meta-analysis. Lancet Neurol. 2011;10(7):626-636. doi:10.1016/S1474-4422(11) 70109-0

4. Huang ZQ, Meng ZH, Hou ZJ, et al. Geometric parameter analysis of ruptured and unruptured aneurysms in patients with symmetric bilateral intracranial aneurysms: a multicenter CT angiography study. Am J Neuroradiol. 2016;37(8):1413-1417. doi:10.3174/ajnr.A4764 
5. Dhar S, Tremmel M, Mocco J, et al. Morphology parameters for intracranial aneurysm rupture risk assessment. Neurosurgery. 2008;63 (2):185-197. doi:10.1227/01.NEU.0000316847.64140.81

6. Jing L, Fan J, Wang Y, et al. Morphologic and hemodynamic analysis in the patients with multiple intracranial aneurysms: ruptured versus unruptured. PLoS One. 2015;10(7):e0132494. doi:10.1371/journal. pone.0132494

7. Singh V, Vignesh S, Neyaz Z, Phadke RV, Mehrotra A, Mishra P. Detection and evaluation of intracranial aneurysms in the posterior fossa by multidetector computed tomography angiography - comparison with digital subtraction angiography. Asian $J$ Neurosurg. 2019;14(2):491. doi:10.4103/ajns.AJNS_290_18

8. Zhang J, Can A, Lai PM, et al. Morphological variables associated with ruptured basilar tip aneurysms. Sci Rep. 2021;11(1):1-9.

9. Liu J, Chen Y, Zhu D, et al. A nomogram to predict rupture risk of middle cerebral artery aneurysm. Neurol Sci. 2021;1-8.

10. Liu Q, Jiang P, Jiang Y, et al. Prediction of aneurysm stability using a machine learning model based on PyRadiomics-derived morphological features. Stroke. 2019;50(9):2314-2321. doi:10.1161/ STROKEAHA.119.025777

11. Ambekar S, Madhugiri V, Bollam P, Nanda A. Morphological differences between ruptured and unruptured basilar bifurcation aneurysms. J Neurol Sci. 2013;74(2):91.

12. Amigo N, Valencia Á. Determining significant morphological and hemodynamic parameters to assess the rupture risk of cerebral aneurysms. J Med Biol Eng. 2019;39(3):329-335. doi:10.1007/ s40846-018-0403-0

13. Detmer FJ, Chung BJ, Jimenez C, et al. Associations of hemodynamics, morphology, and patient characteristics with aneurysm rupture stratified by aneurysm location. Neuroradiology. 2019;61 (3):275-284. doi:10.1007/s00234-018-2135-9
14. Van Der Kolk NM, Algra A, Gabrië'1 JE. Risk of aneurysm rupture at intracranial arterial bifurcations. Cerebrovasc Dis. 2010;30(1):29-35. doi:10.1159/000313441

15. Wang Y, Cheng M, Liu S, et al. Shape related features of intracranial aneurysm are associated with rupture status in a large Chinese cohort. J Neurointerv Surg. 2021:neurintsurg-2021-017452. doi:10.1136/ neurintsurg-2021-017452

16. Ma D, Tremmel M, Paluch RA, Levy EL, Meng H, Mocco J. Size ratio for clinical assessment of intracranial aneurysm rupture risk. Neurol Res. 2010;32(5):482-486. doi:10.1179/ 016164109X12581096796558

17. Sato H, Kamide T, Kikkawa Y, et al. Clinical characteristics of ruptured intracranial aneurysm in patients with multiple intracranial aneurysms. World Neurosurg. 2021;149:e935-e941. doi:10.1016/j. wneu.2021.01.072

18. Lauric A, Baharoglu MI, Malek AM. Ruptured status discrimination performance of aspect ratio, height/width, and bottleneck factor is highly dependent on aneurysm sizing methodology. Neurosurgery. 2012;71(1):38-46. doi:10.1227/NEU.0b013e3182503bf9

19. Meng H, Feng Y, Woodward SH, et al. Mathematical model of the rupture mechanism of intracranial saccular aneurysms through daughter aneurysm formation and growth. Neurol Res. 2005;27 (5):459-465. doi:10.1179/016164105X25171

20. Zhang M, Peng F, Tong X, et al. Associations between haemodynamics and wall enhancement of intracranial aneurysm. Stroke Vasc Neurol. 2021:svn-2020-000636. doi:10.1136/svn-2020-000636

21. Neyazi B, Swiatek VM, Skalej M, et al. Rupture risk assessment for multiple intracranial aneurysms: why there is no need for dozens of clinical, morphological and hemodynamic parameters. Ther Adv Neurol Disord. 2020;13:1756286420966159. doi:10.1177/ 1756286420966159
International Journal of General Medicine

\section{Publish your work in this journal}

The International Journal of General Medicine is an international, peer-reviewed open-access journal that focuses on general and internal medicine, pathogenesis, epidemiology, diagnosis, monitoring and treatment protocols. The journal is characterized by the rapid reporting of reviews, original research and clinical studies across all disease areas. The manuscript management system is completely online and includes a very quick and fair peer-review system, which is all easy to use. Visit http://www.dovepress.com/ testimonials.php to read real quotes from published authors. 